Accelerator Division

Alternating Gradient Synchrotron Department

BROOKHAVEN NATIONAL LABORATORY

Upton, New York 11973

Accelerator Division

Technical Note

AGS/AD/Tech. Note No. 380

\title{
TRANSVERSE INSTABILITY WITH LARGE SPACE CHARGE TUNE SHIFT
}

M. Blaskiewicz and W.T. Weng

September 8, 1993 


\title{
TRANSVERSE INSTABILITY WITH LARGE SPACE CHARGE TUNE SHIFT
}

\author{
M. Blaskiewicz and W. T. Weng
}

\section{ABSTRACT}

The problem of transverse instabilities in circular machines with a large space charge tune shift is considered. In particular, we consider the situation where the space charge tune spread is large compared to the synchrotron tune. The different synchrotron modes couple together, yielding a more complicated situation than when the magnitude of the betatron tune shift is small compared to the synchrotron tune. As the space charge tune shift grows it is found that the coherent frequencies of different modes can cross, without dramatic increases in growth rates. In the extreme limits where the synchrotron frequency vanishes, or where the synchrotron frequency is large compared to any frequency shifts, we find that the space charge tune spread can lead to Landau damping. A new technique for the intermediate range is also presented.

\section{INTRODUCTION}

The problem of transverse instabilities in bunched beams has received much attention in the past. In the low intensity limit, where the magnitude of the betatron tune shift is small compared to the synchrotron tune, well established formalisms exist $[1,2,3,4,5]$. These formalisms yield expressions for the betatron frequency shifts and the normal modes of the beam. When the space charge tune shift is large compared to the synchrotron tune, the low intensity formulae usually yield frequency shifts that are larger than the synchrotron frequency, which violates the assumptions in the derivation of the equations. The large frequency shifts are due to the transverse space charge impedance which is given by,

$$
Z_{\perp, \text { S.C. }}(\omega)=i \frac{R Z_{0}}{\beta^{2} \gamma^{2}}\left(\frac{1}{a^{2}}-\frac{1}{b^{2}}\right),
$$

where $R$ is the machine radius, $\beta=v / c, \gamma$ is the Lorentz factor, $Z_{0}=377 \Omega, a$ is the radius of a uniform equivalent beam, and $b$ is the radius of the vacuum chamber. When $a \ll b$, the frequency shifts obtained from the low intensity formulae are essentially weighted averages of the incoherent space charge frequency shift. When the betatron tune shift is comparable to, or larger than, the synchrotron tune, the mode coupling formalism has been used $[6,7,8,4,5]$. The treatments, in this moderate coupling limit, usually include the coupling between only a few synchrotron modes, as the equations become very complicated when a large number of modes are included. Studies in the moderate coupling limit have usually been applied to high energy electron machines, where the transverse impedance is thought to be dominated by parasitic modes in the accelerating cavities. These parasitic modes produce a resonator impedance of the form,

$$
Z(\omega)=\frac{R_{\perp}}{\frac{\omega}{\omega_{r}}+i Q_{r}\left(1-\frac{\omega^{2}}{\omega_{r}^{2}}\right)}
$$

where $R_{\perp}$ is the transverse shunt impedance of the resonator, $Q_{r}$ is the quality factor, and $\omega_{r}$ is the angular resonant frequency. For a broad band resonator impedance, it is generally found that the growth rate of the most unstable mode 
increases dramatically when the current rises above the threshold where the coherent frequencies of two neighboring modes cross. This is referred to as the mode coupling instability.

This paper considers the transverse stability problem when the space charge tune shift is large. In section II, the equations used in the moderate coupling limit are reviewed, and an upper limit to the growth rate for a Gaussian beam. is derived. We consider the simplest, non-trivial case of mode coupling analytically and find that the space charge inpedance may effect the threshold of the instability, but that the magnitude of the growth rate is limited by the I'sistive impedance.

In section III, the effect of the space charge tune spread is considered. First, the case of an unperturbed beam и ith constant radius, parabolic line density, and the associated incoherent space charge frequency spread is considered. T'he Vlasov equation is reduced to a matrix equation for this case. Solution of the lowest order synthetic kernel approximation, in the weak coupling limit, shows that incoherent space charge may lead to Landau damping if the broad band transverse impedance is large enough. Next, we consider the moderate coupling case where the space cnarge tune spread is a few times larger than the synchrotron tune. When the unperturbed distribution is Gaussian, t.le pertubation can be expanded in Hermite polynomials and the differential equation can be reduced to a matrix equation. The system is solved by truncating the matrix and numerically solving the eigensystem. It is found that the mode frequencies can cross, without creating instability, even when some resistance is present. Finally, we consider the limit where the synchrotron motion is neglected. In this limit, the incoherent space charge force may be handled exactly. The eigenvalue problem is solved for the case where the impedance consists of a single narrow band resonator, and space charge. It is found that space charge can dramatically reduce growth rates, and even damp them completely. Clur conclusions are summarized in section $\mathrm{V}$.

\section{MODERATE COUPLING}

In this section, first order perturbation theory on the Vlasov equation is used to obtain a dispersion relation for the transverse dipole modes. We begin by considering the equations of motion when intensity dependent effects are neglected.

For the longitudinal motion let $\theta$ denote $S / R$ where $R$ is the machine radius and $S$ is the longitudinal Frenet-Serret coordinate. The angular revolution frequency is $\omega_{0}$ and

$$
\ddot{\phi}+\frac{\omega_{s}^{2}}{h} \sin (h \phi)=0
$$

vrhere a dot denotes a derivative with respect to time $(t), \phi=\theta-\omega_{0} t$ is angular phase measured with respect to the synchronous coordinates, $\omega_{s}$ is the synchrotron frequency and $h$ is the harmonic number. Let $y$ denote the transverse coordinate of interest. We assume constant lattice functions and neglect horizontal to vertical coupling. The transverse equation of motion is

$$
\ddot{y}+\omega_{y}^{2}[1+(\xi-\eta) \delta]^{2} y=\frac{\dot{y} \dot{\delta}(\xi-\eta)}{1+(\xi-\eta) \delta}
$$

vihere $\omega_{y}$ is the angular betatron frequency for an on momentum particle, $\eta$ is the frequency slip factor, $\xi$ is the chromaticity, and $\delta$ is the fractional momentum deviation. For horizontal motion, one could include the momentum i.duced width by making the substitution $y \rightarrow y-\delta R / \gamma_{t}^{2}$ in equation (4), but we will not. The fractional momentum c.eviation is related to the longitudinal coordinate via $\dot{\phi}=-\omega_{0}\left[\eta \delta+O\left(y^{2} / R^{2}\right)\right]$, and we neglect the correction that clepends on $y$.

Let $\Psi(y, \dot{y}, \phi, \dot{\phi}, t)$ denote the phase space density. There are $M$ bunches in the ring which are identical for no instability. Assume a solution of the form

$$
\Psi_{\delta}=\sum_{k=0}^{M-1}\left\{\Psi_{0}(\phi-2 \pi k / M, \dot{\phi}, y, \dot{y})+e^{2 \pi i k s / M-i \Omega t} \Psi_{1}(\phi-2 \pi k / M, \dot{\phi}, y, \dot{y})\right\}
$$


where $s=0,1, \ldots M-1$ is the coupled bunch mode number and $\Psi_{1}$, which depends on $s$, is small compared to $\Psi_{0}$. Only the real part of the distribution has physical meaning. The transverse coherent force is driven by the dipole moment of the beam. Any resonating structure with a fixed position in the ring will be driven by the the dipole moment as a function of azimuth and time, $D(\theta, t)$. The normalization is defined by $D(\theta, t)=\langle y(\theta, t)\rangle \rho(\theta, t)$ where $\langle y(\theta, t)\rangle$ is the average offset of the beam and $\rho(\theta, t)$ is the line density of the particles. For the solution of the Vlasov equation, the natural longitudinal coordinate is $\phi$, since the distribution oscillates only with frequency $\Omega$ in this coordinate. If we define the dipole eigenfunction in the beam frame by

$$
D(\phi)=\int d \dot{\phi} d y d \dot{y} \Psi_{1}(\phi, \dot{\phi}, y, \dot{y}) y
$$

then the dipole moment in the ring frame is given by

$$
\begin{aligned}
D(\theta, t) & =\int_{0}^{2 \pi} d \phi D(\phi) e^{-i \Omega t} \delta\left(\theta-\phi-\omega_{0} t\right) \\
& =\sum_{k} D_{k} e^{i(k M+s)\left(\theta-\omega_{0} t\right)-i \Omega t} \\
& =D(\phi) e^{-i \Omega t}
\end{aligned}
$$

where $D_{k}$ is the Fourier component of $D(\phi)$ for harmonic $k M+s$. In addition to the coherent force there is also an incoherent intensity dependent force which produces the incoherent space charge tune depression. This will be neglected for now and considered in section III. The coherent force is related to the dipole moment using a transverse wake potential in the smooth approximation,

$$
\begin{aligned}
F_{\perp}(\theta, y, t) & =\frac{q^{2} \omega_{0}}{2 \pi R} \int_{0}^{\infty} d \tau W_{\perp}(\tau) D(\theta, t-\tau) \\
& =\frac{i q^{2} \omega_{0}^{2}}{2 \pi c} \sum_{k=-\infty}^{\infty} D_{k} Z_{\perp}\left[(k M+s) \omega_{0}+\Omega\right] e^{i(k M+s) \phi-i \Omega t}
\end{aligned}
$$

where $q$ is the charge on a single particle, $W_{\perp}$ is the wake potential, and $Z_{\perp}$ is the transverse impedance. Notice that the force in the second expression is given in the beam frame coordinates and oscillates only at frequency $\Omega$ there. However, in the ring frame, all the betatron side bands drive the impedance. The expression for $D_{k}$ may be obtained by integrating over a single bunch,

$$
D_{k}=\frac{M}{2 \pi} \int_{-\pi / M}^{\pi / M} D(\phi) e^{-i(k M+s) \phi} d \phi .
$$

The problem is reduced to studying the behavior of a single bunch, which we take to be centered at $\phi=0$.

Consider the amplitude angle variables defined implicitly by

$$
\begin{aligned}
y & =A \cos \chi \\
\dot{y} & =-\omega_{\perp}(\delta) A \sin \chi \\
\phi & =r \cos \psi \\
\dot{\phi} & =-\omega_{s} r \sin \psi .
\end{aligned}
$$

The longitudinal variables follow from the equations of motion with the approximation $\sin (h \phi) \approx h \phi$. The transverse variables are approximate, they satisfy the constraint that the amplitude of the $y$ oscillation does not depend on time, which follows from equation (4). The unperturbed distribution is given by $\Psi_{0}=L_{0}(r) T_{0}(A)$. The normalization in the 
$\mathrm{n}$ əw variables is taken as

$$
\begin{aligned}
\int_{0}^{\infty} L_{0}(r) r d r & =\frac{1}{2 \pi}, \\
\int_{0}^{\infty} T_{0}(A) A d A & =\frac{N_{t}}{2 \pi M},
\end{aligned}
$$

where $N_{t}$ is the total number of particles in the ring and we have absorbed factors of the oscillation frequencies. The Vlasov equation in first order perturbation theory is approximated by

$$
-i \Omega \Psi_{1}+\omega_{\perp}(\delta) \frac{\partial \Psi_{1}}{\partial \chi}+\omega_{s} \frac{\partial \Psi_{1}}{\partial \psi}+\dot{A} \frac{\partial \Psi_{0}}{\partial A}=0 .
$$

The momentum dependence of the betatron frequency is taken to first order and is given by

$$
\omega_{\perp}(\delta)=\omega_{y}+r Q_{y} \omega_{s}\left(\frac{\xi}{\eta}-1\right) \sin \psi
$$

where $Q_{y}$ is the tune of an on momentum particle. The coherent force is present in $\dot{A}$ which is give by

$$
\dot{A}=-\frac{\sin \chi}{\gamma m \omega_{y}} F_{\perp}(r \cos \psi),
$$

where the time dependence has been divided out of $F_{\perp}(\phi)$, and the momentum dependence of the betatron frequency is $\mathrm{n}, \mathrm{t}$ included in the coherent force. Next, we approximate the solution of equation $(10)$ as $\Psi_{1}=g_{1}(r, \psi) \exp (i \chi) d T_{0} / d A$ which amounts to neglecting the coupling between the betatron side bands and choosing the upper sideband $\Omega \approx \omega_{y}$. The accompanying approximation is to take $\sin \chi \approx-i \exp (i \chi) / 2$, in $\dot{A}$. The equation for the distribution becomes

$$
-i \Omega g_{1}+i \omega_{\perp}(\delta) g_{1}+\omega_{s} \frac{\partial g_{1}}{\partial \psi}=\frac{-i}{2 \gamma m \omega_{y}} F_{\perp}(\phi) L_{0}(r)
$$

The coherent force is given by equation (8) with

$$
D_{k}=-\frac{N_{t}}{2 \pi} \int_{0}^{\infty} r d r \int_{0}^{2 \pi} d \psi g_{1}(r, \psi) e^{-i(k M+s) r \cos \psi}
$$

At this point all the transverse variables have been removed and we are left with an equation which involves the lc ngitudinal variables alone. Next, the momentum dependence of the betatron frequency is removed by substituting $g_{l}=g_{2} \exp \left[i Q_{y}(\xi / \eta-1) r \cos \psi\right]$ which results in,

$$
-i\left(\Omega-\omega_{y}\right) g_{2}+\omega_{s} \frac{\partial g_{2}}{\partial \psi}=\frac{-i e^{-i Q_{y}(\xi / \eta-1) r \cos \psi}}{2 \gamma m \omega_{y}} F_{\perp}(\phi) L_{0}(r) .
$$

Iividing equation (15) by $\omega_{s}$ yields an equation of the form

$$
i Q g_{2}(r, \psi)+\frac{\partial g_{2}}{\partial \psi}=\tilde{F}(r, \psi)
$$

where $\tilde{F}$ is equal to the right hand side of equation(15) divided by $\omega_{s}$, and $Q=\left(\omega_{y}-\Omega\right) / \omega_{s}$. The boundary condition is that $g_{2}$ is a periodic function of $\psi$. The solution of equation (16) is given by,

$$
g_{2}(r, \psi)=\frac{1}{e^{2 \pi i Q}-1} \int_{0}^{2 \pi} d \psi^{\prime} e^{i Q \psi^{\prime}} \tilde{F}\left(r, \psi+\psi^{\prime}\right)
$$

which is easily verified by direct substitution. At this point one can concentrate on solving for $g_{2}$ or on solving for the dipole harmonics. The final result will be identical in either case, and we will take the second option [4]. Given the 
expression (17) for $g_{2}$, and the relationship of $g_{1}$ to $g_{2}$, equation (14) is used to transform the integral equation into a matrix equation. The kernel is simplified using the Bessel generating function,

$$
e^{i x \sin \theta}=\sum_{k=-\infty}^{\infty} J_{k}(x) e^{i k \theta}
$$

The final result is,

$$
D_{m}=\sum_{n=-\infty}^{\infty} T_{m n} D_{n}
$$

where the matrix is given by,

$$
T_{m n}=\frac{i c q \bar{I} Z_{\perp}\left[(n M+s) \omega_{0}+\Omega\right]}{2 E_{T} \omega_{s} Q_{y}} \int_{0}^{\infty} r d r L_{0}(r) \sum_{\mu=-\infty}^{\infty} \frac{1}{Q+\mu} J_{\mu}(\tilde{n} r) J_{\mu}(\tilde{m} r)
$$

In equation (19) $E_{T}=\gamma m c^{2}, \bar{I}$ is the average (DC) current, $\tilde{n}=n M+s-Q_{y}(\xi / \eta-1)$ and $\tilde{m}$ is defined in the same way as $\tilde{n}$. Note that

$$
Q=\left(\omega_{y}-\Omega\right) / \omega_{s},
$$

contains the coherent frequency and plays the part of an eigenvalue.

At this point we will consider the special case of a gaussian unperturbed distribution,

$$
L_{0}(r)=\frac{e^{-r^{2} / 2 \sigma^{2}}}{2 \pi \sigma^{2}} .
$$

For this case, the integral in equation (19) can be found in standard tables [9][p 710]. The expression for the matrix element becomes

$$
\begin{aligned}
T_{m n} & =i \chi_{n}(\Omega) e^{-\left(\tilde{m}^{2}+\tilde{n}^{2}\right) \sigma^{2} / 2} \sum_{\mu=-\infty}^{\infty} \frac{I_{\mu}\left(\tilde{n} \tilde{m} \sigma^{2}\right)}{Q+\mu} \\
\chi_{n}(\Omega) & =\frac{c q \bar{I} Z_{\perp}\left[(n M+s) \omega_{0}+\Omega\right]}{4 \pi E_{T} \omega_{s} Q_{y}} .
\end{aligned}
$$

We know of no exact solutions to equation (21) and will need to use various approximations in solving it. Before proceeding with the approximations, we establish an upper limit on the magnitude of the coherent frequency shift. Starting with the matrix equation we find

$$
\left|D_{m}\right| \leq \sum_{n}\left|T_{m n}\right|\left|D_{n}\right| \leq \sup _{k}\left|D_{k}\right| \sum_{n}\left|T_{m n}\right|
$$

Where we assume that the spectrum of dipole harmonics is bounded, so that its supremum is finite. It follows that,

$$
\begin{aligned}
\frac{\left|D_{m}\right|}{\sup _{k}\left|D_{k}\right|} & \leq \sum_{n}\left|T_{m n}\right| \\
& \leq \sum_{n}\left|\chi_{n}\right| e-\left(\tilde{m}^{2}+\tilde{n}^{2}\right) \sigma^{2} / 2 \sum_{\mu=-\infty}^{\infty} \frac{I_{\mu}\left(\left|\tilde{n} \tilde{m} \sigma^{2}\right|\right)}{|Q+\mu|} \\
& \leq \frac{\sup _{n}\left|\chi_{n}\right|}{\inf _{\mu}|Q+\mu|} \sum_{n} e^{-\left(\tilde{m}^{2}+\tilde{n}^{2}\right) \sigma^{2} / 2+\left|\sigma^{2} \tilde{m} \tilde{n}\right|}
\end{aligned}
$$

where we have used a summation theorem for the modified Bessel functions [9]. Since,

$$
e^{-\left(x^{2}+y^{2}\right)+2|x y|} \leq e^{-(x+y)^{2}}+e^{-(x-y)^{2}}
$$


fc I real $x$ and $y$, the right hand side of the inequality can be made independent of $m$. Taking the supremum of the left $h$ ind side over $m$ and simplifying gives

$$
\inf _{\mu}|Q+\mu| \leq \sup _{n}\left|\chi_{n}\right| 2 \sum_{n} e^{-n^{2} M^{2} \sigma^{2} / 2} \approx \sup _{n}\left|\chi_{n}\right| \frac{\sqrt{8 \pi}}{\sigma M} .
$$

In practice, inequality (25) may be used to obtain a quick upper limit on growth rates in Gaussian beams. More a!curate estimates require approximate solutions and are considered next.

\section{A. Weak Coupling Limit}

Before proceeding to the mode coupled case it will be instructive to examine the low intensity limit. In this limit, $\left|\lambda_{: n}\right| \ll \sigma M$ for all $n$. Therefore, the solution requires that $|Q+\mu| \approx 0$ for some $\mu$, and that $\Omega \approx \omega_{y}+\mu \omega_{s}$. This a.?proximation is used for $\Omega$ in the evaluation of the impedance. Only the term in the sum with the small denominator is kept. Additionally, the modified Bessel function is approximated by its leading order term,

$$
I_{\mu}\left(\tilde{n} \tilde{m} \sigma^{2}\right) \approx \frac{\left(\tilde{n} \tilde{m} \sigma^{2}\right)^{|\mu|}}{2^{|\mu|}|\mu| !}
$$

When equation (26) is substituted into equation (21) one finds that $D_{m}=(\tilde{m} \sigma)^{|\mu|} \exp \left(-\tilde{m}^{2} \sigma^{2} / 2\right)$ is the eigenvector. $\mathrm{S}$ abstituting this solution and solving for the coherent frequency gives,

$$
\Omega=\omega_{y}+\mu \omega_{s}-\frac{i \omega_{s}}{2^{|\mu|}|\mu| !} \sum_{n} \chi_{n}\left(\omega_{y}+\mu \omega_{s}\right) e^{-\tilde{n}^{2} \sigma^{2}}(\tilde{n} \sigma)^{2|\mu|} .
$$

Usually, the offset by $\mu \omega_{s}$ in the evaluation of the impedance is negligible and one uses $\chi_{n}\left(\omega_{y}\right)$. For the cases of interest, the frequency shift is large enough that including the synchrotron offset is misleading. Expression (27) is the same as t] lat obtained using the lowest order Besnier polynomial expansion [7] and is essentially the same as the expression given by Sacherer [1].

Higher order modes must be included when approximation (26) is not justified. This is the case when there is a significant impedance at frequencies higher than $\omega_{0} / \sigma$ and needs to be considered even if the frequency shift is sunall compared to the synchrotron tune. For this case, one still retains a single value of $\mu$ but includes higher order terms in the expansion for the Bessel function. Such a treatment is justified in studies of longitudinal stability where the frequency shift is small compared to the synchrotron tune, and space charge contributes to the high frequency irapedance. However, it is not justified for the transverse case when $\left|\chi_{n}\right| \gtrsim 1$. The latter is of particular interest and will be studied in the following.

\section{Fi. Solution With Moderate Coupling}

VThen $\left|\chi_{n}\right| \gtrsim 1$, a few values of $\mu$ can significantly contribute to the sum. For this case it is advantageous to rearrange the sum involving the Bessel functions,

$$
\sum_{\mu} \frac{I_{\mu}\left(\tilde{n} \tilde{m} \sigma^{2}\right)}{Q+\mu}=\sum_{\ell=0}^{\infty} a_{\ell}(Q)\left(\tilde{n} \tilde{m} \sigma^{2}\right)^{\ell}
$$

Inı general,

$$
a_{\ell}(Q)=\sum_{\mu=-\ell}^{\ell} \frac{1}{\mu+Q} \frac{1}{2^{\ell}[(\ell-\mu) / 2] ![(\ell+\mu) / 2] !}
$$

where the sum is over even $\mu$ if $\ell$ is even and over odd $\mu$ is $\ell$ is odd. The first few coefficients are given by

$$
a_{0}(Q)=\frac{1}{Q}, \quad a_{1}(Q)=\frac{Q}{Q^{2}-1}, \quad a_{2}(Q)=\frac{1}{2} \frac{Q^{2}-2}{Q\left(Q^{2}-4\right)}
$$


To proceed with the solution, the sum on the right hand side of equation (28) is truncated at $\ell=\ell_{\max }$. Substituting this expression into equation (21) implies that $D_{m}$ may be written as a linear combination

$$
D_{m}=\sum_{\ell=0}^{\ell_{m a x}} \alpha_{\ell}(\tilde{m} \sigma)^{\ell} e^{-\tilde{m}^{2} \sigma^{2} / 2}
$$

where the $\alpha_{\ell}$ are unknown coefficients. Defining the force coefficients

$$
f_{\ell}=i \sum_{n} \chi_{n}\left(\omega_{y}\right)(\tilde{n} \sigma)^{\ell} e^{-\tilde{n}^{2} \sigma^{2}}
$$

the equation for the coherent frequency and eigenvector is given by

$$
\alpha_{p}=a_{p}(Q) \sum_{\ell=0}^{\ell=\ell_{\max }} \alpha_{\ell} f_{\ell+p} .
$$

Analytic solution of equation (33) is possible for $\ell_{\max } \leq 1$. For $\ell_{\max }=0$ one recovers the $\mu=0$ synchrotron mode in the low intensity limit. For $\ell_{m a x}=1$ there are two coupled equations,

$$
\left(\begin{array}{cc}
\left(a_{0} f_{0}-1\right) & a_{0} f_{1} \\
a_{1} f_{1} & \left(a_{1} f_{2}-1\right)
\end{array}\right)\left(\begin{array}{c}
\alpha_{0} \\
\alpha_{1}
\end{array}\right)=0
$$

The determinant of the matrix must vanish for a non-trivial solution. Substituting the expressions for $a_{0}$ and $a_{1}$ from equation (30), one obtains a polynomial constraint on $Q$,

$$
\left(Q-f_{0}\right)\left(Q^{2}-1-Q f_{2}\right)-Q f_{1}^{2}=0 .
$$

The solutions depend on the magnitude and phase of the force coefficients.

For electron machines, the mode coupling is found to be nearly independent of chromaticity [8] and the usual approach is to set $\boldsymbol{\xi}=0$ in the mode coupling formula. Additionally, the source of impedance is thought to be broad band and the characteristic frequency of the bunch spectrum is large compared to the bunching frequency. This allows the summation in equation (32) to be replaced by an integral. Under these circumstances, the force coefficients for even $k$ are real and proportional to the imaginary part of the transverse impedance. For odd $k, f_{k}$ is imaginary and proportional to the real part of the impedance. Consider equation (34) under these circumstances. If $f_{1}=0$ the solutions are given by $Q=f_{0}, f_{2} / 2 \pm \sqrt{1+f_{2}^{2} / 2}$. The solutions for $Q$ are real, even though they can cross as the force coefficients become large. Generally, when $f_{0}, f_{2}$, and $f_{1}^{2}$ are real, $|\operatorname{Im}(Q)| \leq\left|f_{1}\right|$, which is proved in Appendix A. The maximum growth rate is proportional to the resistance, but the instability threshold depends on the reactance as well.

For many applications, the values of $\chi_{n}$ need to be computed numerically. Also, the dispersion relation for $Q$ becomes very complicated as $Q$ increases. Computer code has been written to evaluate the various sums and to solve equation (33) for $\ell_{\max } \leq 4$. As an example consider the case of space charge and a broad band resonator with $Q_{r}=1$, $\omega_{r}=\omega_{0} / \sigma$ and a shunt impedance such that $f_{1}=i 0.2$. Plots of the real part of the resonant frequencies as a function of the average space charge tune shift are shown in Figures 1 through 4 . The onset of the instability occurs when two neighboring curves meet. The threshold value of space charge tune shift is relatively insensitive to $\ell_{\max }$. However, as tune shift increases, the solutions can switch between stable and unstable several times. The detailed behavior of the solutions depends strongly on $\ell_{m a x}$ but the peak growth rates, as a function of space charge tune shift, are usually less sensitive to $\ell_{\max }$.

When the chromaticity, discreteness, etc. are taken into account the solutions always have an imaginary part, but the growth rate can depend strongly on the space charge tune shift. By examining the behavior of the growth rate as a function of tune shift it appears that some useful upper limits can be found, but no threshold information. We go on to consider a more complete physical model which includes threshold information. 


\section{.II. EFFECT OF SPACE CHARGE TUNE SPREAD}

Ir section II, equation (11) was used for the incoherent betatron frequency. In the presence of a large space charge tr.ne spread this equation needs to be modified,

$$
\omega_{\perp}(\delta, \phi)=\omega_{y}+r Q_{y} \omega_{s}\left(\frac{\xi}{\eta}-1\right) \sin \psi-\Delta \omega_{S C} \rho(\phi) / \rho(0),
$$

where $\Delta \omega_{S C}$ is the space charge frequency depression in the center of the bunch, $\rho(\phi)$ is the line density, and it has bı:en assumed that the radius of the beam is independent of $\phi$. Under these conditions, equation (13) becomes

$$
-i \Omega g_{1}+i \omega_{\perp}(\delta, \phi) g_{1}+\omega_{s} \frac{\partial g_{1}}{\partial \psi}=\frac{-i}{2 \gamma m \omega_{y}} F_{\perp}(\phi) L_{0}(r)
$$

\section{A. Parabolic Line Density}

Ir. general, it is difficult to obtain a matrix equation when the betatron frequency is given by equation (35). For a pirrabolic line density with $|\phi| \leq \hat{\phi}$,

$$
\rho(\phi)=\frac{3}{4 \hat{\phi}}\left[1-\frac{\phi^{2}}{\hat{\phi}^{2}}\right],
$$

scme simplification is possible. The transverse betatron frequency is given by,

$$
\omega_{\perp}(\delta, \phi)=\omega_{y}+r Q_{y} \omega_{s}\left(\frac{\xi}{\eta}-1\right) \sin \psi-\Delta \omega_{S C}\left(1-\phi^{2} / \hat{\phi}^{2}\right) .
$$

The $\psi$ dependence of the betatron frequency is removed from equation (36) by the substitution

$$
\begin{aligned}
g_{1} & =g_{2} \exp \left[i Q_{y}\left(\frac{\xi}{\eta}-1\right) r \cos \psi-i \frac{\Delta \omega_{S C}}{4 \hat{\phi}^{2} \omega_{s}} r^{2} \sin (2 \psi)\right] \\
& =g_{2} \exp (i \Phi(r, \psi)) .
\end{aligned}
$$

The equation for $g_{2}$ is given by

$$
-i\left[\Omega-\omega_{y}+\Delta \omega_{S C}\left(1-r^{2} / 2 \hat{\phi}^{2}\right)\right] g_{2}+\omega_{s} \frac{\partial g_{2}}{\partial \psi}=\frac{-i e^{-i \Phi(r, \psi)}}{2 \gamma m \omega_{y}} F_{\perp}(\phi) L_{0}(r)
$$

with,

$$
L_{0}(r)=\frac{3}{2 \pi \hat{\phi}^{2}}\left(1-\frac{r^{2}}{\hat{\phi}^{2}}\right)^{1 / 2}
$$

To solve equation (39), let

$$
g_{2}(r, \psi)=\sum_{m} R_{m}(r) \exp (i m \psi)
$$

N.ultiplying by $\exp (-i \mu \psi)$ and integrating over $\psi$ yields,

$$
(Q(r)+\mu) R_{\mu}(r)=2 \pi L_{0}(r) \int_{0}^{\hat{\phi}} x d x \sum_{m} R_{m}(x) \sum_{n} i \chi_{n}(\Omega) K_{\mu}\left(\tilde{n} r, \lambda r^{2}\right) K_{m}\left(\tilde{n} x, \lambda x^{2}\right),
$$

where

$$
\begin{aligned}
K_{\mu}(x, y) & =e^{i \pi \mu / 2} \sum_{p} J_{\mu+2 p}(x) J_{p}(y) \\
\lambda & =\frac{\Delta \omega_{S C}}{4 \omega_{s} \hat{\phi}^{2}}, \\
Q(r) & =\frac{\omega_{y}-\Delta \omega_{S C}\left(1-r^{2} / 2 \hat{\phi}^{2}\right)-\Omega}{\omega_{s}} .
\end{aligned}
$$


Next we expand the kernel in equation (41) as

$$
\sum_{n} i \chi_{n}(\Omega) K_{\mu}\left(\tilde{n} r, \lambda r^{2}\right) K_{m}\left(\tilde{n} x, \lambda x^{2}\right)=\sum_{j, k} \alpha_{j, k}^{\mu, m} f_{j}^{\mu}(r) f_{k}^{m}(x)
$$

where $f_{k}^{m}(r)$ for $k=0,1, \ldots$ form an orthonormal basis on $(0, \hat{\phi})$ with the weighting function $2 \pi r L_{0}(r)$, for each superscript $m$. A natural set of expansion functions are given by

$$
f_{k}^{m}(r)=\left(\frac{2^{m+2+1 / 2}}{3 h_{k, m}}\right)^{1 / 2}\left(\frac{r}{\hat{\phi}}\right)^{m} P_{k}^{m, 1 / 2}\left(1-2 r^{2} / \hat{\phi}^{2}\right)
$$

where $P_{k}^{m, 1 / 2}(x)$ is a Jacobi polynomial and,

$$
h_{k, m}=\frac{2^{m+3 / 2} \Gamma(k+m+1) \Gamma(k+3 / 2)}{(2 k+m+3 / 2) k ! \Gamma(k+m+3 / 2)} .
$$

Defining

$$
b_{k, m}=\int_{0}^{\hat{\phi}} x d x R_{m}(x) f_{k}^{m}(x)
$$

yields a matrix equation given by

$$
b_{p, \mu}=\sum_{m, k} b_{k, m} \sum_{j} \alpha_{j, k}^{\mu, m} \int_{0}^{\hat{\phi}} r d r \frac{2 \pi L_{0}(r) f_{p}^{\mu}(r) f_{j}^{\mu}(r)}{Q(r)+\mu} .
$$

Equation (44) simplifies in the low intensity limit, where $K_{m}\left(\tilde{n} r, \lambda r^{2}\right) \approx e^{i m \pi / 2} J_{m}(\tilde{n} r)$. For this case, the coefficients in the expansion of the kernel can be expanded as

$$
\alpha_{j, k}^{\mu, m}=\sum_{n} i \chi_{n} e^{i(\mu-m) \pi / 2} \tilde{f}_{j}^{\mu}(\tilde{n} \hat{\phi}) \tilde{f}_{k}^{m}(\tilde{n} \hat{\phi})
$$

where

$$
\tilde{f}_{k}^{m}(x)=\frac{\Gamma(k+3 / 2)}{k !}\left(\frac{2^{m+4+1 / 2} 3}{\pi h_{k, m}}\right)^{1 / 2} \frac{j_{m+2 k+1}(x)}{x}
$$

and $j_{n}(x)$ is the spherical Bessel function. In practice, the sums in equation (44) need to be truncated. The simplest case is to take the case where all the summation indicies are zero. Additionally, we will use equation (45). This leads to a dispersion relation of the form

$$
\begin{aligned}
1 & =\hat{\alpha} \int_{0}^{1} \frac{3 x d x \sqrt{1-x^{2}}}{-x^{2}+2\left(\Omega-\omega_{\perp}(0)\right) / \Delta \omega_{S C}} \\
\hat{\alpha} & =\frac{8}{5}\left(1-\frac{a^{2}}{b^{2}}\right)-\frac{2 \omega_{s}}{\Delta \omega_{S C}} \sum_{n} i \hat{\chi}_{n}(\Omega)\left|\frac{3 j_{1}(\tilde{n} \hat{\phi})}{\tilde{n} \hat{\phi}}\right|^{2},
\end{aligned}
$$

where $a$ is the beam radius, $b$ is the pipe radius, and $\hat{\chi}_{n}(\Omega)$ is $\chi_{n}(\Omega)$ calculated without the space charge contribution. A stability diagram for this dispersion relation is shown in Figure 5. The system is stable if $\hat{\alpha}$ lies to the left of the curve.

\section{B. Gaussian Line Density}

When the space charge tune spread is larger than the synchrotron tune, several synchrotron modes can couple. Solving equation (44) is quite difficult so we will present a different approach which is easier to program. Consider equation (36) 
и ith a Gaussian line density. We make the substitution $g_{1}=g_{2} \exp \left[i Q_{y}(\xi / \eta-1) \phi\right]$ and express the result in normalized, C'artesian variables $z=\phi / \sqrt{2} \sigma, v=\dot{\phi} / \sqrt{2} \sigma \omega_{s}$,

$$
\begin{aligned}
-Q g_{2}(z, v) & +\frac{\Delta \omega_{S C}}{\omega_{s}}\left\{g_{2} e^{-z^{2}}-\left(1-a^{2} / b^{2}\right) \frac{e^{-\left(z^{2}+v^{2}\right)}}{\sqrt{\pi}} \int_{-\infty}^{\infty} d v_{1} g_{2}\left(z, v_{1}\right)\right\} \\
& +i\left\{v \frac{d g_{2}}{d z}-z \frac{d g_{2}}{d v}\right\}=-i \frac{e^{-\left(z^{2}+v^{2}\right)}}{\pi} \sum_{k} \hat{\chi}_{k} e^{i \tilde{k} \sqrt{2} \sigma z} \int d z_{1} d v_{1} e^{-i \tilde{k} \sqrt{2} \sigma z_{1}} g_{2}\left(z_{1}, v_{1}\right)
\end{aligned}
$$

where $\tilde{k}=k M+s+Q_{y}(1-\xi / \eta), \hat{\chi}_{k}$ is $\chi_{k}$ calculated without the space charge contribution, $a$ is the beam radius, $b$ is tile pipe radius, and $Q=\left(\omega_{y}-\Omega\right) / \omega_{s}$ as before. To solve equation (49) expand $g_{2}$ as

$$
g_{2}(z, v)=\sum_{n, m} a_{n, m} H_{n}(z) H_{m}(v) e^{-\left(z^{2}+v^{2}\right)}
$$

where

$$
H_{m}(x)=e^{x^{2}}\left(\frac{d}{d x}\right)^{m} e^{-x^{2}}
$$

is the Hermite polynomial of order $m$ and the sum is over all pairs of non-negative integers. Substituting equation (50) ij equation (49), multiplying by $H_{p}(z) H_{q}(v) d z d v$ and integrating yields a matrix for the expansion coefficients

$$
Q a_{p, q}=\sum_{n, m} T_{p, q, n, m} a_{n, m}
$$

T'he matrix element is given by

$$
\begin{aligned}
T_{p, q, n, m} & =i\left\{n \delta_{m+1}^{q} \delta_{n-1}^{p}-m \delta_{m-1}^{q} \delta_{n+1}^{p}\right\} \\
& +\frac{\Delta \omega_{S C}}{\omega_{s}} \frac{\delta_{m}^{q}\left[1-\delta_{m}^{0}\left(1-a^{2} / b^{2}\right)\right]}{p ! \sqrt{2 \pi}}\left(\frac{i}{\sqrt{2}}\right)^{p-n} \Gamma\left(\frac{n+p+1}{2}\right) \operatorname{EVEN}(n+p) \\
& +i \frac{\delta_{m}^{0} \delta_{q}^{0}}{p !}\left(\frac{i}{\sqrt{2}}\right)^{p-n} \sum_{k} \hat{\chi}_{k}(\sigma \tilde{k})^{n+p} e^{-\tilde{k}^{2} \sigma^{2}}
\end{aligned}
$$

where $\delta_{n}^{m}$ is the Kroneker delta, $\Gamma(x)=(x-1)$ !, and $\operatorname{EVEN}(k)$ is 1 when $k$ is even and zero otherwise.

In real machines, transverse instabilities are often expected and damping systems are installed. The effect of a linear feedback damping system can be included in the Vlasov equation and additional terms to the $T_{p, q, n, m}$ matrix are obtained. For a damper which acts on rigid modes at a rate $\alpha_{d, 0}$ the addition to the matrix is

$$
\delta_{0} T_{p, q, n, m}=\frac{i \alpha_{d, 0}}{\omega_{s}} \delta_{q}^{0} \delta_{m}^{0} \frac{\tilde{q}^{p+n} i^{p-n}}{p ! 2^{p}} e^{-\tilde{q}^{2} / 2}
$$

where $\tilde{q}=\sqrt{2} \sigma Q_{y}(1-\xi / \eta)$. Similar expressions for head-tail and higher order dampers are easily obtained.

In practical applications the sum in equation (51) needs to be truncated. The sum will be truncated by taking t:rms with $0 \leq m+n \leq \ell_{\max }$ which allows for a nearly direct comparison between the Hermite expansion method and tine method used in section IIB. For $\ell_{\max }=0$ one obtains the $\mu=0$ weak coupling frequency shift with the proviso tinat the space charge impedance be given by,

$$
Z_{\text {vall,S.C. }}(\omega)=-i \frac{R Z_{0}}{b^{2} \beta^{2} \gamma^{2}}
$$

When $\ell_{\max }=1$ the characteristic equation is,

$$
\left(f_{0}-Q\right)\left[\left(f_{2}-Q\right)(\epsilon / \sqrt{2}-Q)-1\right]-f_{1}^{2}(\epsilon / \sqrt{2}-Q)=0 .
$$


where $f_{k}$ is defined by equation (32), with equation (54) used for the space charge impedance, and $\epsilon=\Delta \omega_{s C} / \omega_{s}$. In the limit $\Delta \omega_{S C} \rightarrow 0$, equation (55) is the same as equation (34). Additionally, in the broad band limit appropriate to electron machines, $|\operatorname{Im}(Q)| \leq\left|f_{1}\right|$ as was true for equation (34).

When $\ell_{\max }$ is large, numerical techniques must be employed, and computer code has been written to do so. As an example, consider the case with the same broad band resonator as was used in Figures 1 through 4 . Plots of the real part of $Q$ as a function of $\Delta \omega_{S C} / \omega_{s}$ are show in Figures 6 through 9. Since space charge satisfies $\Delta \omega_{S C} / \omega_{s}=-\sqrt{2} f_{0}$, the limits on the horizontal axes are essentially identical in Figures 1 through 4 and Figures 6 through 9 . There are several differences between the two sets of figures.

One obvious difference is that Figures 1 through 4 show a tendency for $R e(Q)$ to decrease as the space charge tune shift becomes larger, while the opposite is true for Figures 6 through 9 . This difference is largely due to the fact that the incoherent betatron tune was assumed constant for Figures 1 through 4, while space charge tune depression was included in the Hermite expansion. A closer agreement between the two methods could be found by taking the intensity dependence of the incoherent betatron tune into account for the moderate coupling case. However, this modification would have no effect on the growth rates predicted using equation (33).

Another difference, which is not as apparent from the figures, is that the growth rates for the two cases were different. For the Hermite expansion, instability was not predicted until $\ell_{\max }=3$, and the peak growth rates for $\ell_{\max }=3$ and $\ell_{\max }=4$ were nearly identical. For $\ell_{\max }=5$ the peak growth rate was somewhat smaller.

As long as the space charge tune depression is not too large it has been found that the characteristics of the most unstable mode appear to approach their actual values for reasonable values of $\ell_{m a x}$ in the Hermite expansion. For some machines $\Delta \omega_{S C} \gtrsim 100 \omega_{s}$ and calculation using the Hermite expansion becomes a large scale computational problem. We go on to consider another approach designed for this large tune shift limit.

\section{Strong Coupling Case}

For the strong coupling case, $\Delta \omega_{S C} \gg \omega_{s}$. Instead of trying to include the motion of a very large number of modes, we will neglect the synchrotron motion altogether, giving $\dot{\phi}=0$ and a beam that is frozen longitudinally. The vertical equation of motion is given by

$$
\ddot{y}+\omega_{y}^{2} y=\frac{F_{\perp}(x, y, \phi, t)}{\gamma m}
$$

where both transverse coordinates appear in the collective force. The collective force contains contributions due to the currents flowing in the walls of the vacuum chamber and direct particle-particle forces which are responsible for the space charge tune spread. We average equation (56) over the particles at a fixed value of $\phi$. The direct particle-particle forces cancel when averaged over the cross section, since they come from a Coulomb-like potential. We approximate the wall induced forces using the transverse impedance, as in the earlier sections. This results in an eigenvalue problem for $\langle y(\phi, t)\rangle=\langle y(\phi)\rangle \exp (-i \Omega t)$, which is given by

$$
-\Omega^{2}<y(\phi)>+\omega_{y}^{2}<y(\phi)>=\frac{F_{w a l l}(\phi)}{\gamma m} .
$$

The force due to the currents in the wall is given by equation (8) the space charge impedance given by equation (54).

To turn equation (57) into a proper eigenvalue problem we approximate $\omega_{y}^{2}-\Omega^{2}=2 \omega_{y}\left(\omega_{y}-\Omega\right)$ and set $\Omega=\omega_{y}$ in the argument of the impedance. Multiplying the result by the line density gives,

$$
\left(\omega_{y}-\Omega\right) D(\phi)=\frac{\rho(\phi)}{2 \omega_{y} \gamma m} F_{w a l l}(\phi),
$$

where $\rho(\phi)$ is the line density of the particles, and $\omega_{y}$ is the bare betatron frequency for an on momentum particle. As a consistency check, note that setting $\omega_{s}=0$ in equation (36) leads to equation (58) which, since equation (58) is quite simple, supports equation (36). We proceed to solve this problem for a model system. 
Consider a system with space charge and a single narrow band resonator. It is assumed that the impedance of t.xe resonator is negligible compared to space charge for all but a single value of $n=K$. Under these circumstances equation (58) becomes

$$
\delta \Omega D(\phi)=-\hat{\rho}(\phi)\left[\Delta D(\phi)+i \hat{R} D_{K} e^{i(K M+s) \phi}\right],
$$

where $\delta \Omega=\Omega-\omega_{y},-i \Delta / \hat{R}=Z_{w a l l, S C} / R, R$ is the resonator impedance when $n=K$, and $\hat{\rho}(\phi)$ is the normalized line density. Solving for $D(\phi)$ and extracting the $K$ th harmonic leads to a dispersion relation,

$$
1=-\frac{i M \hat{R}}{2 \pi} \int_{-\pi / M}^{\pi / M} \frac{\hat{\rho}(\phi) d \phi}{\delta \Omega+\Delta \hat{\rho}(\phi)} .
$$

For a constant line density with $\hat{\rho}(\phi)=1 / 2 \hat{\phi}$ for $|\phi| \leq \hat{\phi}$, the integral is trivial with the result $\delta \Omega=-i M \hat{R} / 2 \pi-\Delta / 2 \hat{\phi}$. The growth rate is independent of the reactance. On the other hand, if $\hat{\rho}(\phi)=\cos ^{2}(\pi \phi / 2 \hat{\phi}) / \hat{\phi}$, the frequency shift, calculated in Appendix B, is given by,

$$
\delta \Omega=-\frac{\Delta}{\hat{\phi}}\left[1-\frac{\tilde{R}^{2}}{1+2 i \tilde{R}}\right],
$$

where $\tilde{R}=M \hat{R} \hat{\phi} / \pi \Delta$, and the solution is valid for $|1+2 i \tilde{R}|>1$. Setting $\Delta=0$ gives $\operatorname{Im}\left(\delta \Omega_{0}\right)=-M \hat{R} / 2 \pi$. The actual growth rate is always smaller than the $\Delta=0$ growth rate with the ratio of the actual growth rate to the $\Delta=0$ growth rate given by $\operatorname{Im}(\delta \Omega) / \operatorname{Im}\left(\delta \Omega_{0}\right) \lesssim 2|\tilde{R}|$. The growth rate reduction can be understood by considering the dipole eigenfunction

$$
D(\phi)=\frac{\cos ^{2}(\pi \phi / 2 \hat{\phi}) e^{i(K m+s) \phi}}{\sin ^{2}(\pi \phi / 2 \hat{\phi})-\tilde{R}^{2} /(1+2 i \tilde{R})} .
$$

For small $\tilde{R}$, the magnitude of $D$ is relatively small unless $|\phi| \lesssim \hat{\phi}|\tilde{R}|$. In physical terms, the space charge impedance causes the local coherent betatron frequency to vary along the bunch, like a collection of oscillators with different natural frequencies. The resonator is only partially effective at maintaining the oscillators at a single coherent frequency, r $2 s u l t i n g$ in a reduced growth rate.

\section{$Y$. CONCLUSIONS}

T'he effect of space charge on transverse instabilities has been considered. When the space charge induced tune spread was neglected, it was found that the threshold for transverse mode coupling instability depends on space charge. For the simplest, non-trivial case of 3 modes, an upper limit on the growth rate that depended only on the resistance was fisund. When the space charge tune spread was included, we found that transverse instabilities can be Landau damped in the low intensity limit, but the beam size and transverse impedance constraints are fairly severe. When the space charge tune shift is not much larger than the synchrotron tune an expansion using Hermite polynomials is applicable. ' 'his technique has the added benefit that increasing the number of synchrotron modes is easy and calculations using as many at \pm 10 synchrotron modes have been performed. In the high intensity limit where the synchrotron tune is negligible, we found that the space charge forces can lead to growth rates which are far smaller than those predicted using Besnier polynomial or rigid bunch approximations.

\section{]References}

[1] F.J. Sacherer, CERN/SI-BR/72-5, (1972).

[2] A.W. Chao \& C.Y. Yao, PEP-Note 321, (1979).

[3] J.L. Laclare, CERN 87-03, p 264 (1987).

[4] J.M. Wang, BNL 39057, (1986). 
[5] A.W. Chao Physics of Collective Beam Instabilities in High Energy Accelerators Wiley, 1993.

[6] Y. Chin, K. Satoh \& K. Yokoya, Particle Accelerators 13, p45 (1983).

[7] K. Satoh \& Y. Chin, Nuclear Instruments and Methods 207, p309 (1983).

[8] G. Besnier, D. Brandt \& B. Zotter, CERN LEP-TH/84-11 (1984).

[9] I.S. Gradshteyn \& I.M. Ryzhik Table of Integrals Series and Products Academic Press 1965.

\section{Appendix A}

In this appendix it is shown than the growth rates obtained from equation (34) are bounded via $|\operatorname{Im}(Q)| \leq\left|f_{1}\right|$, when $f_{0}, f_{2}$, and $f_{1}^{2}$ are all real.

Rewrite equation (34) to yield

$$
\left(Q-f_{0}\right)\left(Q-F_{+}\right)\left(Q-F_{-}\right)=Q f_{1}^{2} .
$$

Where $F_{ \pm}=f_{2} / 2 \pm \sqrt{1+f_{2}^{2} / 4}$. Notice that $F_{+}>0$ and $F_{-}<0$. Taking absolute values and using the triangle inequality yields

$$
|\operatorname{Im}(Q)|^{2} \leq\left|Q-f_{0}\right|\left|Q-F_{+}\right|=\frac{\left|Q f_{1}^{2}\right|}{\left|Q-F_{-}\right|} .
$$

Dividing by $Q-F_{+}$instead of $Q-F_{-}$yields

$$
|\operatorname{Im}(Q)|^{2} \leq\left|Q-f_{0}\right|\left|Q-F_{-}\right|=\frac{\left|Q f_{1}^{2}\right|}{\left|Q-F_{+}\right|} .
$$

Since $a \leq b$ and $a \leq c$ implies $a \leq \min (b, c)$,

$$
|\operatorname{Im}(Q)|^{2} \leq\left|f_{1}^{2}\right| \min \left(\frac{|Q|}{\left|Q-F_{-}\right|}, \frac{|Q|}{\left|Q-F_{+}\right|}\right) .
$$

$F_{-}$and $F_{+}$are real, and of opposite sign. When they are viewed as vectors on the complex plane one of them will add constructively to any $Q$ one cares to choose. Hence, one of the denominators on the right hand side will be larger than $|Q|$, giving $|\operatorname{Im}(Q)| \leq\left|f_{1}\right|$.

\section{Appendix B}

The coherent frequency for a cosine squared line density is derived.

Defining $\tilde{Q}=2 \hat{\phi} \delta \Omega / \Delta$, equation (60) becomes

$$
1=-\frac{i \tilde{R}}{2 \pi} \int_{-\pi}^{\pi} d \theta \frac{1+\cos \theta}{\tilde{Q}+1+\cos \theta} .
$$

Making the substitution $z=\exp (i \theta)$, equation (B1) becomes,

$$
\frac{i}{\tilde{R}}=1+\frac{i \tilde{Q}}{\pi} \oint \frac{d z}{2 z(1+\tilde{Q})+z^{2}+1}
$$

where the integral is along the unit circle in the complex plane. The integrand of equation (B2) has simple poles at $z_{ \pm}=-(1+\tilde{Q}) \pm \sqrt{(1+\tilde{Q})^{2}-1}$. Since $z_{+} z_{-}=1$ either both poles lie on the unit circle or one lies inside and the other lies outside. Assume the second case, which is equivalent to assuming a non-singular integrand in equation B1. Define $z_{i n}$ to be the root inside the unit circle and $z_{\text {out }}$ to be the root outside the unit circle. The integral is trivial with the result

$$
1-\frac{i}{\tilde{R}}=\frac{2 \tilde{Q}}{z_{\text {in }}-z_{\text {out }}}
$$


Squaring both sides of equation B3 gives

$$
\left(1-\frac{i}{\tilde{R}}\right)^{2}=\frac{\tilde{Q}^{2}}{\tilde{Q}^{2}+2 \tilde{Q}}
$$

A ssuming $\tilde{Q} \neq 0$ yields

$$
\tilde{Q}=-2 \frac{(1+i \tilde{R})^{2}}{1+2 i \tilde{R}}
$$

w nich is equivalent to equation (61). Equation (B5) suggests that very large frequency shifts are possible if $\tilde{R} \approx i / 2$, but this does not occur. For $|1+2 i \tilde{R}|<1$, equation (B4) is satisfied but equation (B3) is not, and the system is Landau dimped. 


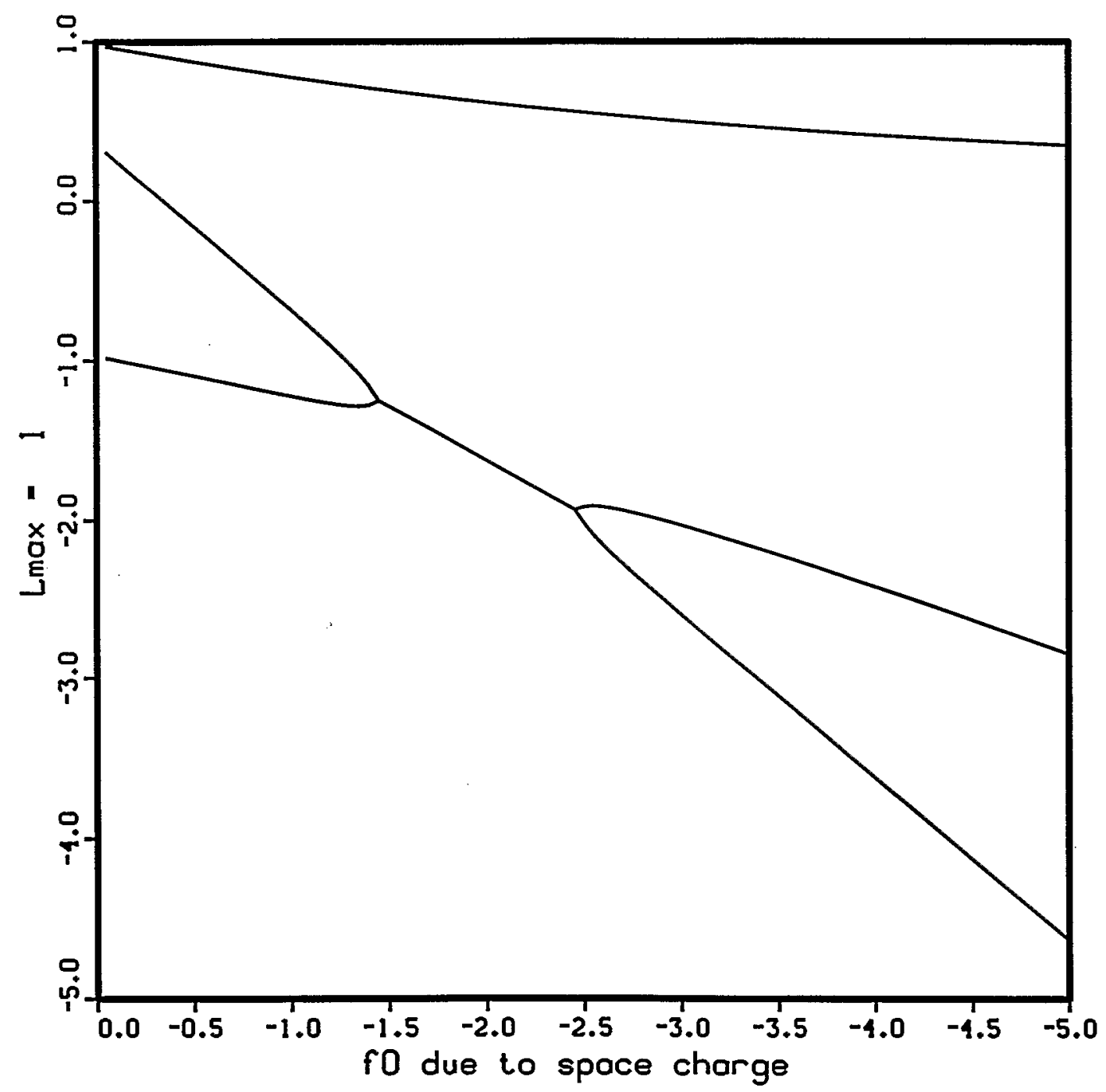

Figure 1. Real part of $Q$ as a function of $f_{0}$ due to space charge for $\ell_{\max }=1$. 


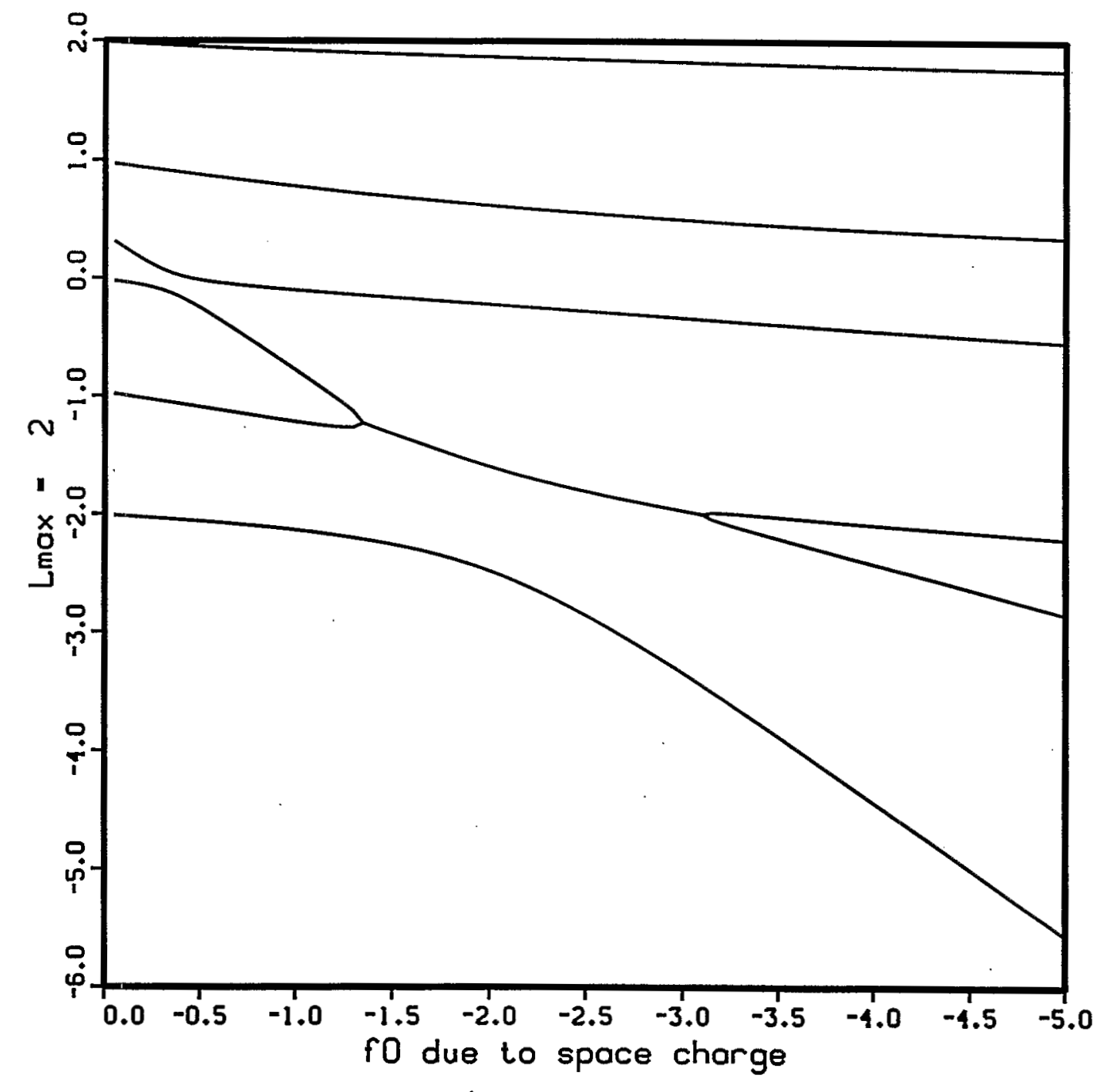

Figure 2. Real part of $Q$ as a function of $f_{0}$ due to space charge for $\ell_{\max }=2$. 


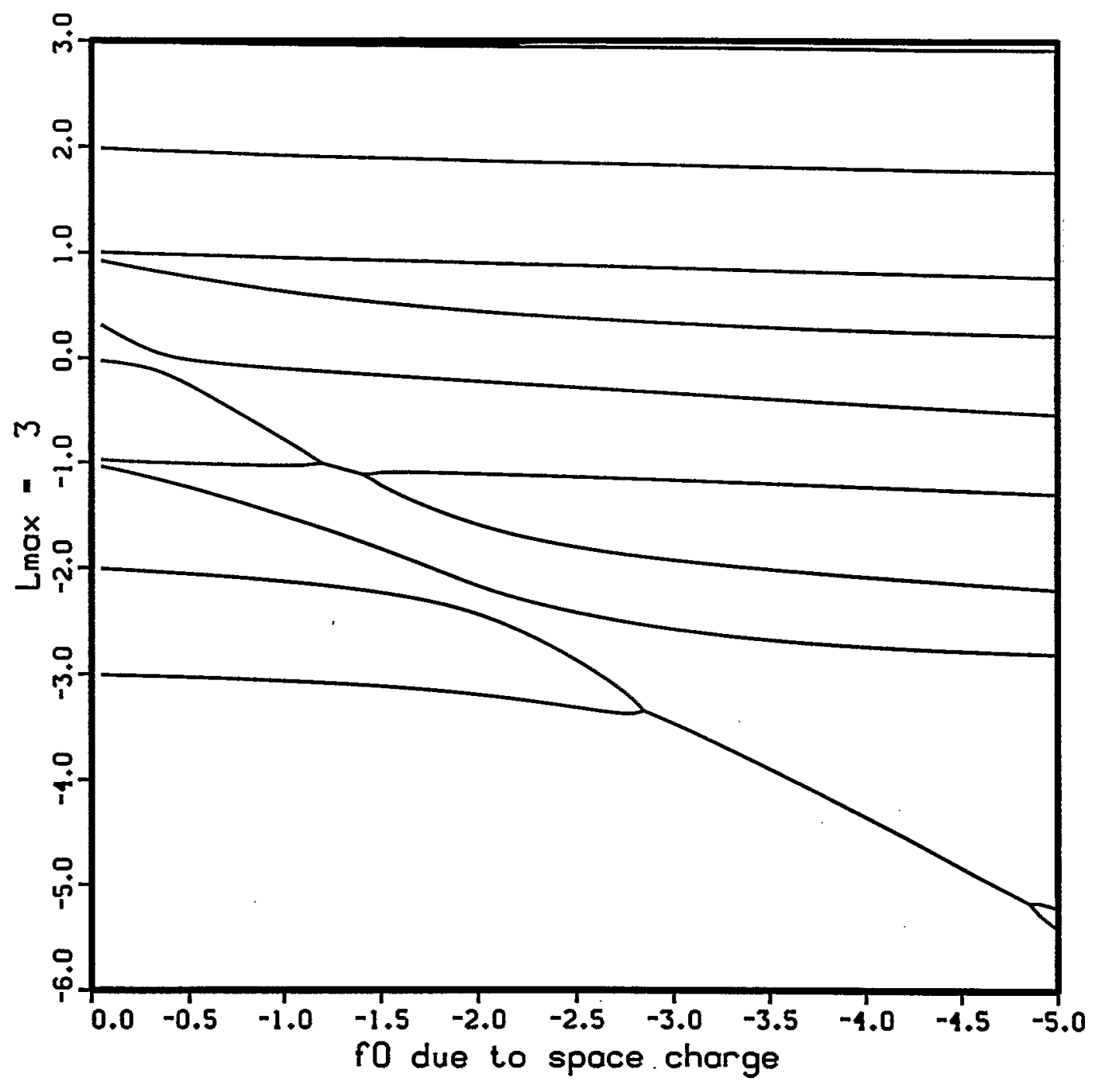

Figure 3. Real part of $Q$ as a function of $f_{0}$ due to space charge for $\ell_{\max }=3$. 


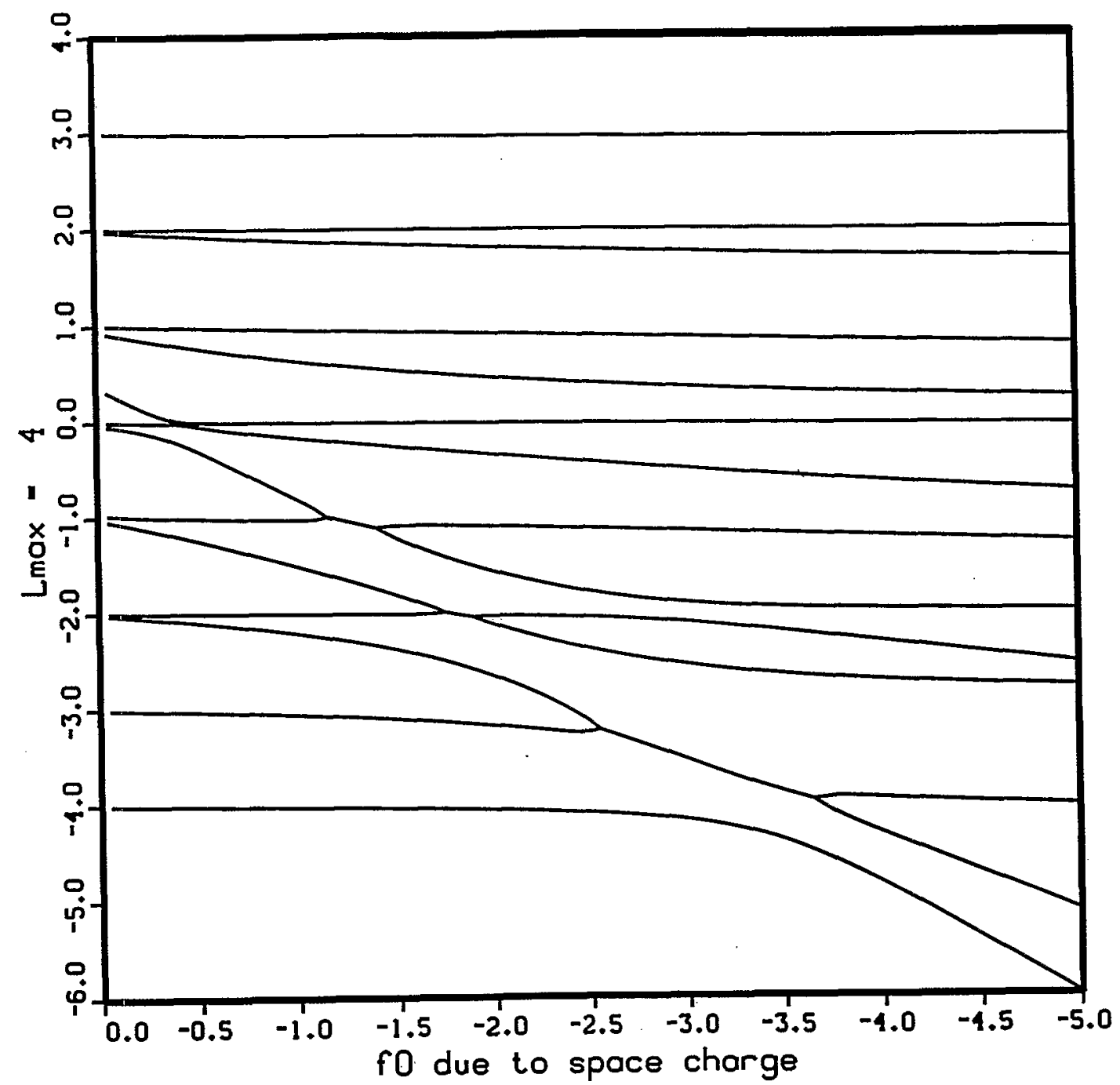

Figure 4. Real part of $Q$ as a function of $f_{0}$ due to space charge for $\ell_{\max }=4$. 


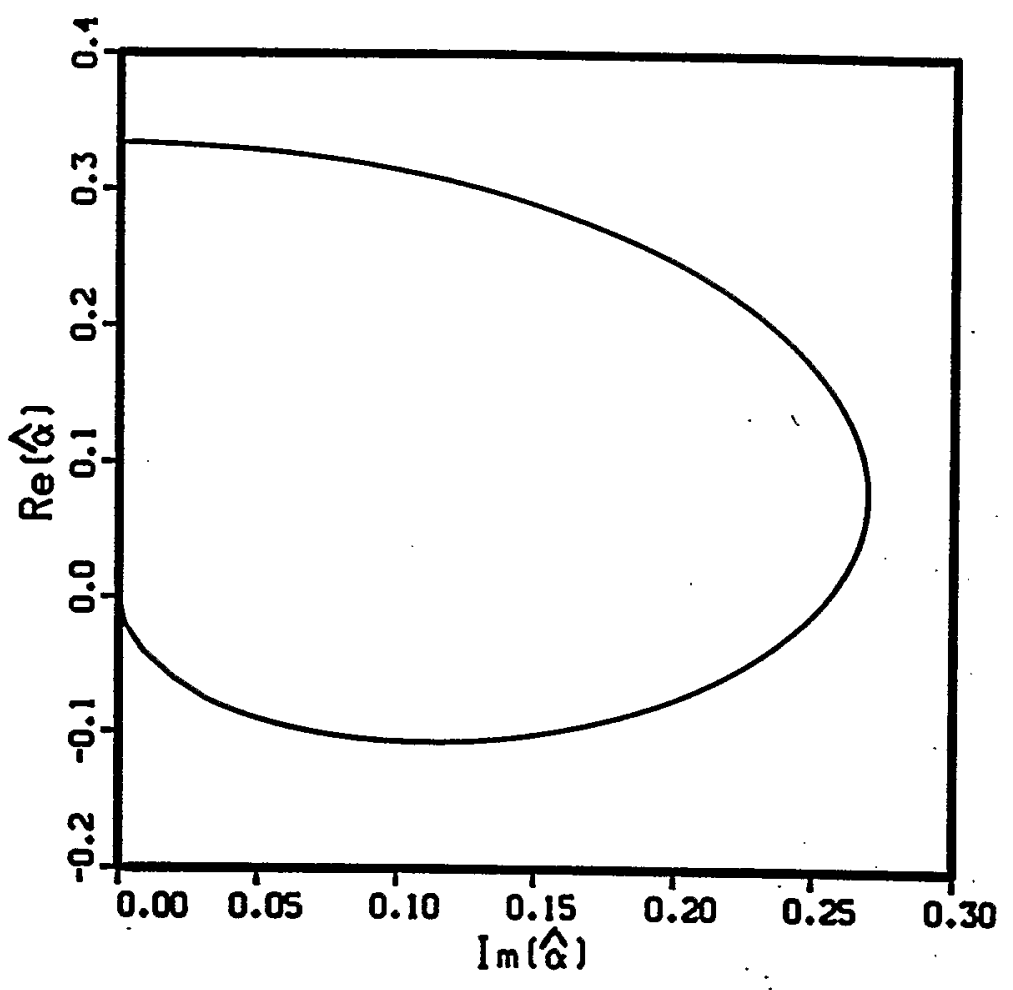

Figure 5. Stability countours from equation (47); points to the left of the contour are stable. 


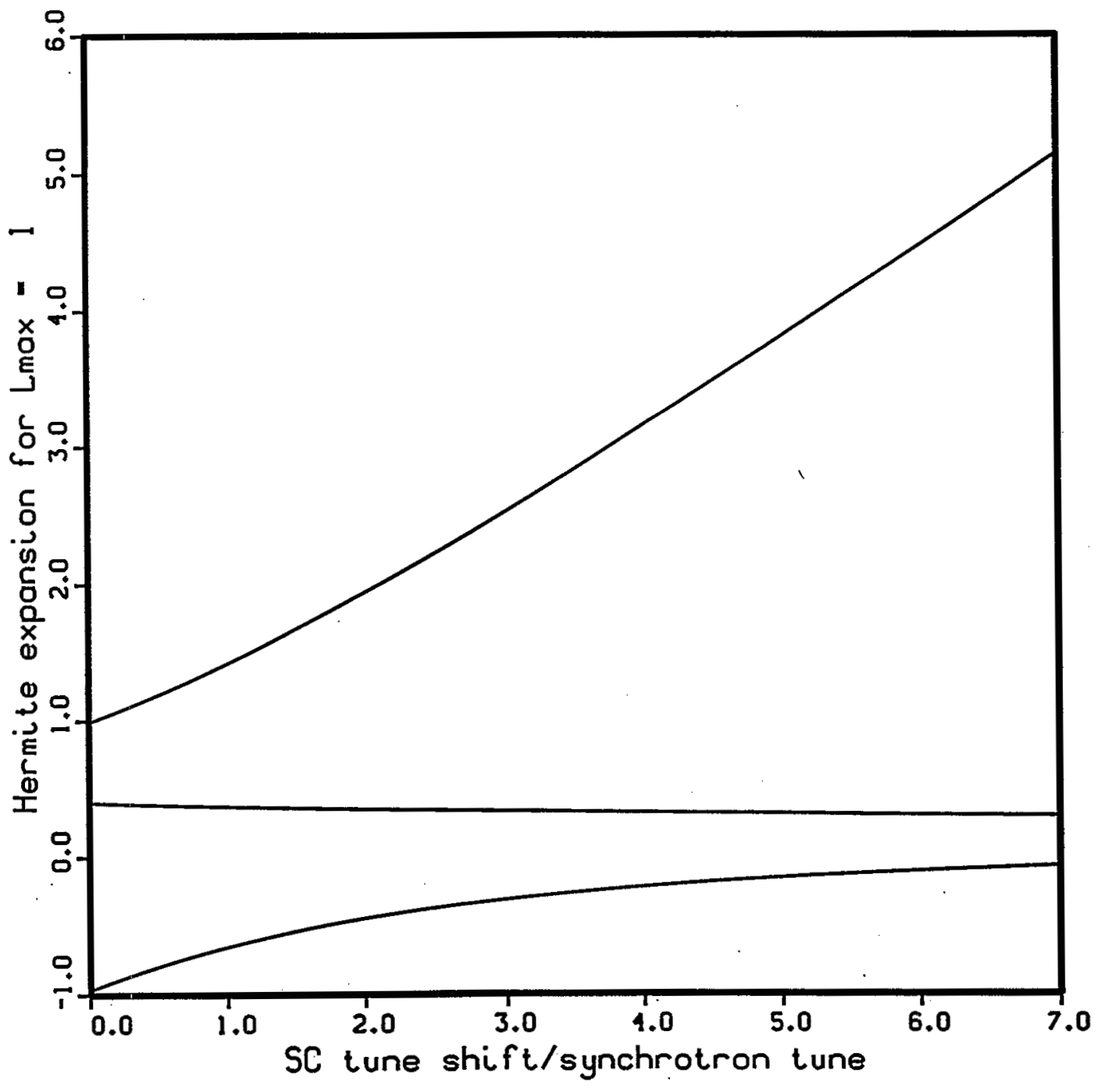

Figure 6. Real part of $Q$ as a function of $\Delta \omega_{S C} / \omega_{s}$ using the Hermite expansion for $\ell_{\max }=1$. 


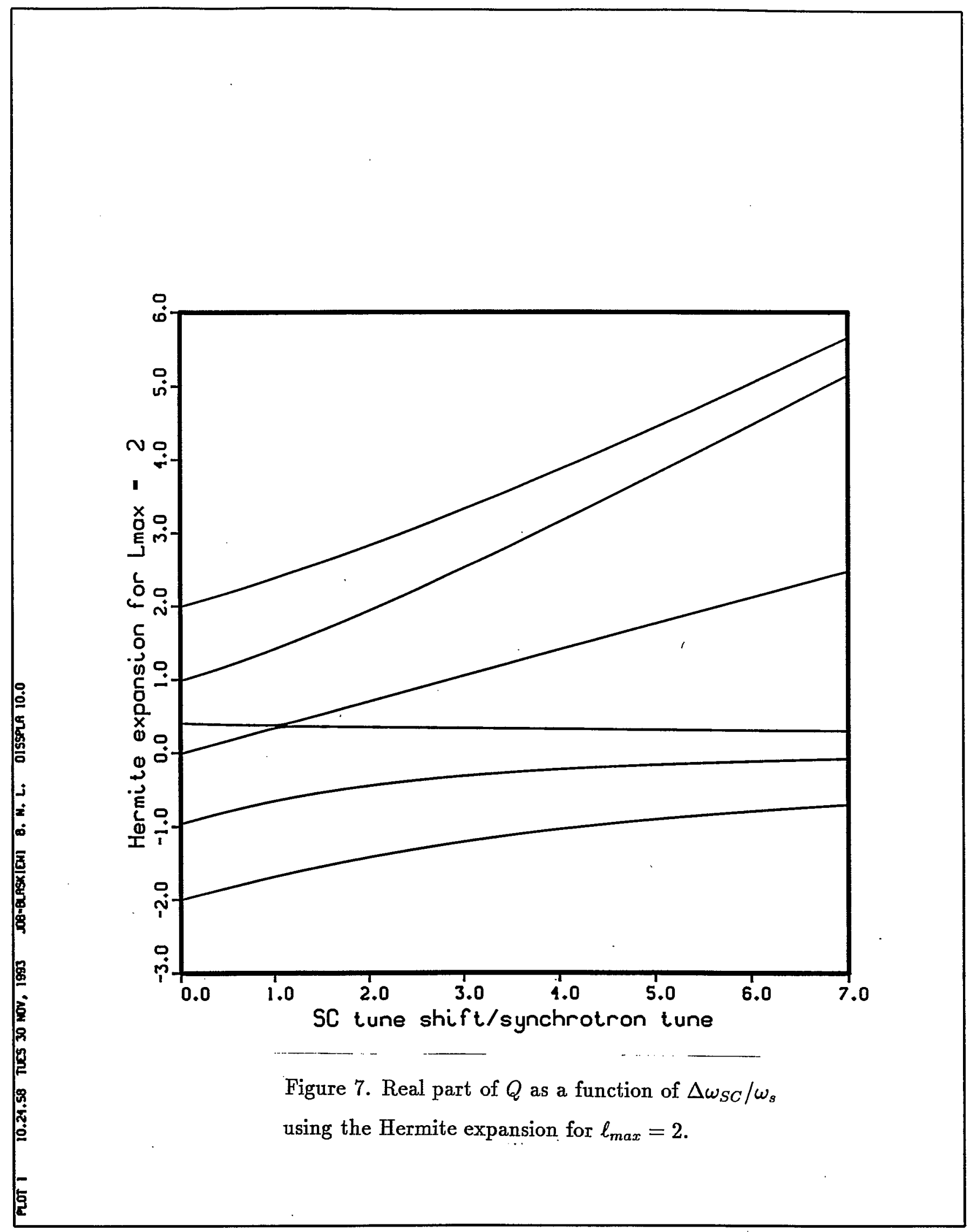




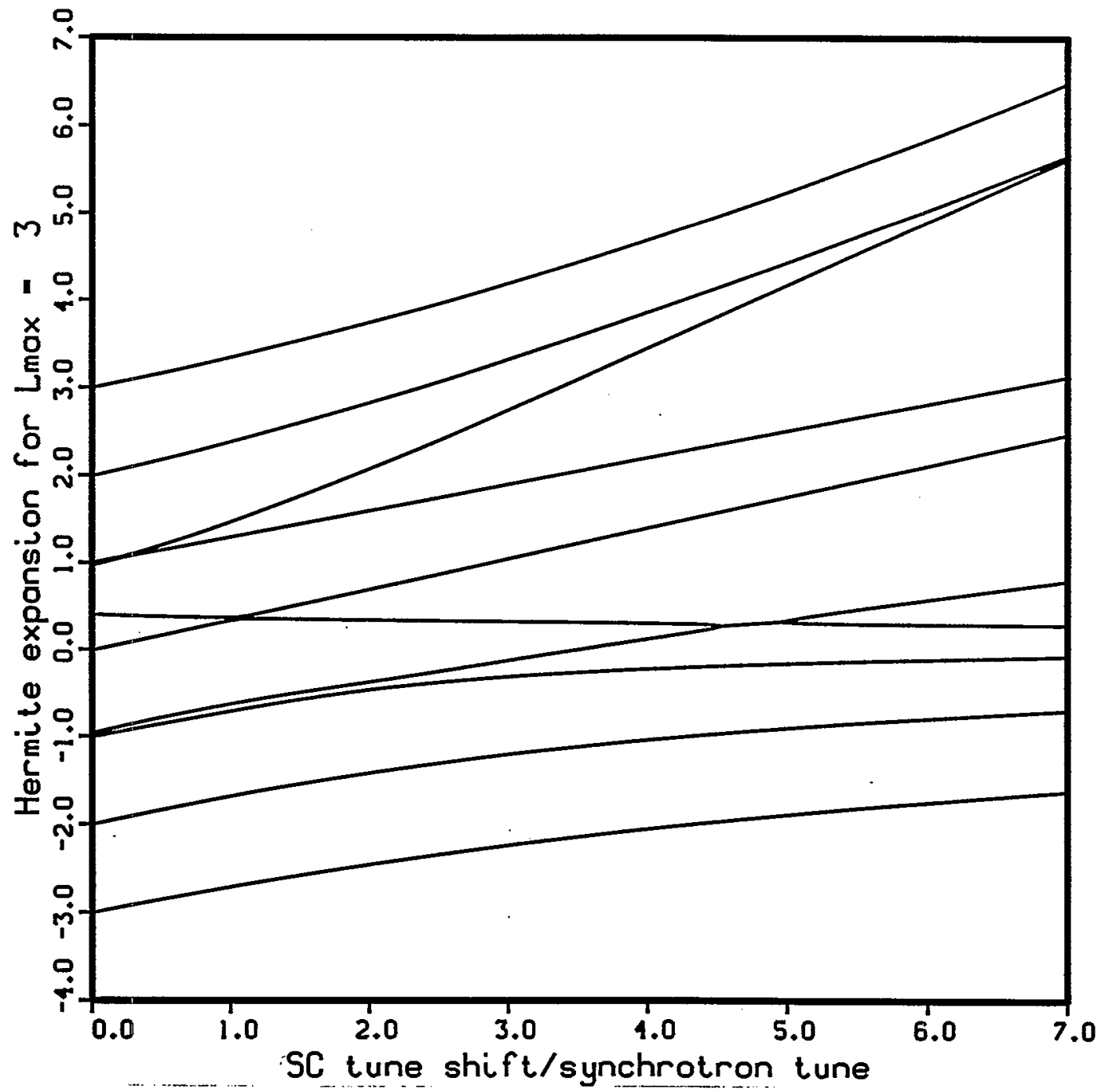

Figure 8. Real part of $Q$ as a function of $\Delta \omega_{S C} / \omega_{s}$ using the Hermite expansion for $\ell_{\max }=3$. 


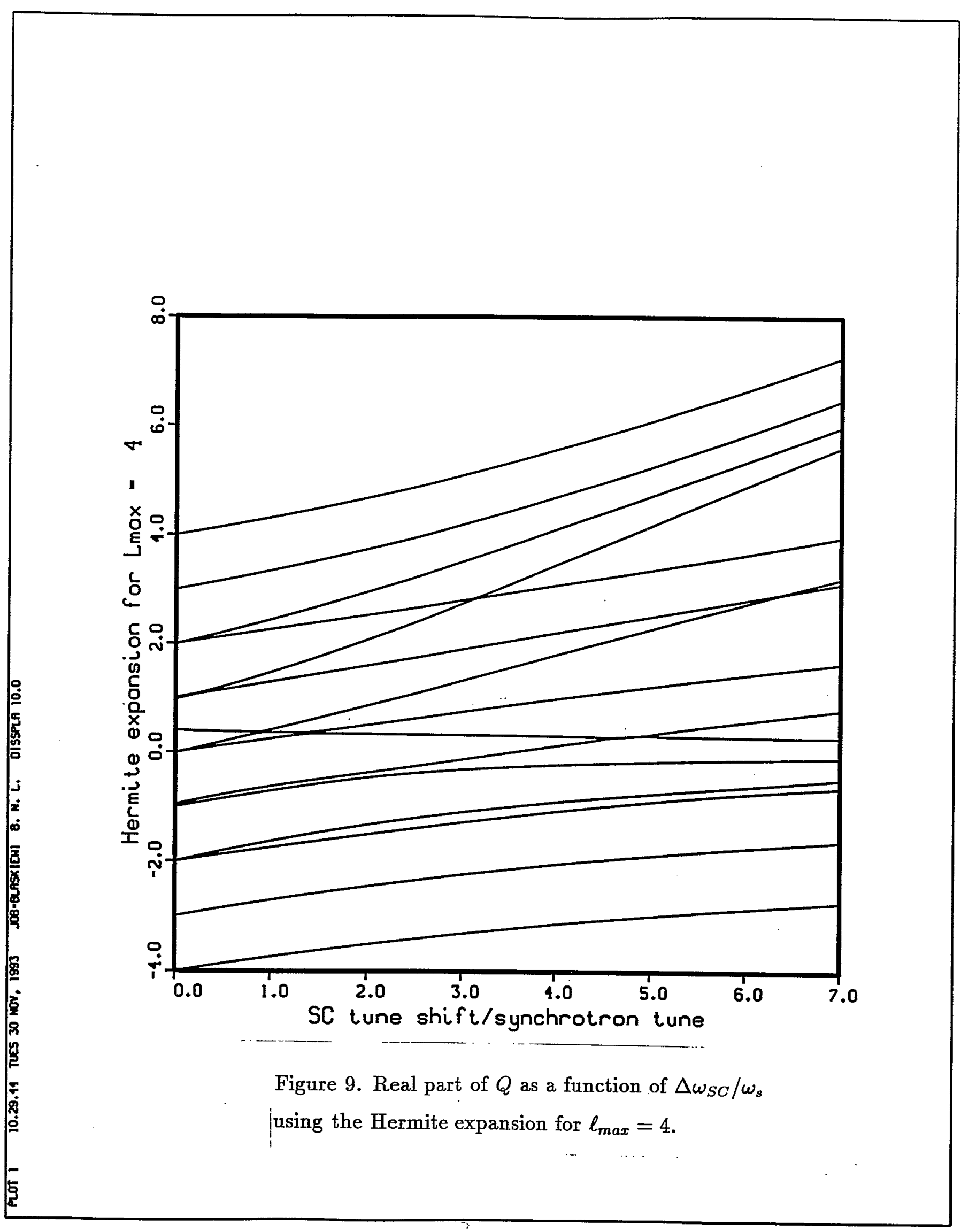

